5. Leuchter AF, Hunter AM, Krantz DE, Cook IA. Rhythms and blues: modulation of oscillatory synchrony and the mechanism of action of antidepressant treatments. Ann N Y Acad Sci. 2015;1344:78-91.

In Reply Fogelson and Leuchter have responded to our proposed staging scheme for treatment-resistant depression (TRD) published in JAMA Psychiatry. ${ }^{1}$ We do not fundamentally disagree with their arguments; however, we stand by our proposal that psychiatry needs to move toward a more empirically based TRD definition.

Fogelson and Leuchter express concern about the lack of evidence to support using different antidepressant classes to improve antidepressant efficacy. In putting forward our preliminary TRD staging model, we included both psychopharmacology and psychotherapy treatments. Further, we describe preliminary treatment recommendations for stage I TRD, which include nonpharmacological treatments (electroconvulsive therapy and repetitive transcranial magnetic stimulation) as possible treatment trials for stage I TRD.

If the treatments are pharmacological, the rationale for requiring failure of 2 different classes is based on existing limited evidence of the predictive value of specific antidepressant trial combinations in predicting future resistance. For example, does failure to benefit from escitalopram and citalopram (same class) convey the same information about future likelihood of benefit as a failed response to 2 medications from different classes, say escitalopram and nortriptyline? We believe there is no compelling evidence to address this question. When suggesting an arbitrary threshold and general guideline for defining TRD, it seems prudent to require 2 different treatment classes, whether within pharmacological interventions or across the different types of intervention.

Existing data suggest the opposite problem. Evidence from a community referral TRD clinic found patients with TRD failed an average of 3.6 selective serotonin reuptake inhibitor class antidepressants, with many failing 5 selective serotonin reuptake inhibitors. ${ }^{2}$ Thus, many patients with TRD fail a multitude of similar mechanism antidepressants for years.

Regarding their other arguments (TRD is likely multifactorial/heterogeneous and TRD models based on number of failed treatment trials would not address subtypes): the authors suggest that patients with TRD and comorbid personality disorder or history of childhood adversity/trauma may require specific antidepressant interventions to be considered resistant. We agree that in the future, TRD staging will need refinement if clinical features/biomarkers that predict outcome with specific antidepressant interventions are determined. However, presently, we do not have this level of knowledge and these associations are not sufficiently robust or reliable to justify the refinements suggested by the authors.

We concur with the ambitious goal of refining specific biomarkers and clinical TRD subtypes. However, until achieved, the severe costs of TRD treatment failure (10\% per annum response rate to standard treatments ${ }^{3}$ and high suicide risk $^{4}$ ) warrant a more practical and utilitarian TRD staging system to allow research and clinical practice to progress.

Finally, Fogelson and Leuchter suggest that by highlighting the number of drug trials as the primary measure to stage
TRD, we perpetuate “a neurochemical approach to TRD...” Our proposed TRD model actually does the opposite. An important goal of our model is to help psychiatrists "think outside the box" of standard neurochemical approaches (using more novel mechanism of action treatments, eg, ketamine, repetitive transcranial magnetic stimulation, and novel psychotherapies) relatively early on in treatment, thereby decreasing the period of dealing with TRD.

\author{
Charles R. Conway, MD \\ Mark S. George, MD \\ Harold A. Sackeim, PhD
}

Author Affiliations: Washington University School of Medicine, St Louis, Missouri (Conway); Medical University of South Carolina, Charleston (George); Ralph H. Johnson VA Medical Center, Charleston, South Carolina (George); Columbia University, New York, New York (Sackeim).

Corresponding Author: Charles R. Conway, MD, Washington University School of Medicine, 660 S Euclid Ave, Campus Box 8134, St Louis, MO 63110 (conwaycr @psychiatry.wustl.edu).

Published Online: May 31, 2017. doi:10.1001/jamapsychiatry.2017.0970

Conflict of Interest Disclosures: Dr Conway has received research support from Bristol-Myers Squibb, the Stanley Medical Research Institute, the National Institute of Mental Health, NeoSync Inc, Cyberonics Inc, The Taylor Family Institute for Innovative Psychiatric Research, The August Busch IV Foundation, and Barnes-Jewish Hospital Foundation. He previously served as a speaker for Bristol-Myers Squibb and Otsuka Pharmaceuticals. He has served as an unpaid consultant to Cyberonics Inc. Dr George has no equity ownership in any device or pharmaceutical company. He does occasionally consult with industry, although he has not accepted consulting fees from anyone who manufactures a transcranial magnetic stimulation device because of his role in National Institutes of Health and Department of Defense/Veterans Administration studies evaluating this technology. His total industry-related compensation per year is less than $10 \%$ of his total university salary. He has consulted (unpaid) for Brainsonix, Brainsway Inc, Cervel/NeoStim, MECTA Corporation, Neuronetics, NeoSync Inc, and Nervive. He has consulted for PureTech Ventures and is a data and safety monitoring board member of Microtransponder. He has received research grant funding or equipment from Brainsway Inc, Cervel/NeoStim, MECTA Corporation, Neuronetics Inc, and NeoSync Inc. He is a journal editor for Elsevier Press. Dr Sackeim has consulted for Brainsway Inc, Cyberonics Inc, Eli Lilly Inc, Magstim Ltd, MECTA Corporation, Neosync Inc, Neuronetics Inc, Neuropace Inc, Novartis Inc, and Pfizer Inc.

1. Conway CR, George MS, Sackeim HA. Toward an evidence-based, operational definition of treatment-resistant depression: when enough is enough. JAMA Psychiatry. 2017;74(1):9-10.

2. Conway CR, Gebara MA, Walker MC, et al. Clinical characteristics and management of treatment-resistant depression. J Clin Psychiatry. 2015;76(11): 1569-1570.

3. Dunner DL, Rush AJ, Russell JM, et al. Prospective, long-term, multicenter study of the naturalistic outcomes of patients with treatment-resistant depression. J Clin Psychiatry. 2006;67(5):688-695.

4. Amital D, Fostick L, Silberman $A$, Beckman $M$, Spivak B. Serious life events among resistant and non-resistant MDD patients. $J$ Affect Disord. 2008;110(3): 260-264.

\section{Are There Differences in Disruptions of Reward Processing Between Substance Use Disorder and Gambling Disorder?}

To the Editor To our knowledge, functional magnetic resonance imaging (fMRI) studies to date have reported both striatal hypoactivations and hyperactivations during anticipation and outcome notification of monetary rewards, making it impossible to fit the results to one specific theory of addiction. To clarify these contradicting findings, Luijten et $\mathrm{al}^{1}$ used meta-analyses of fMRI data to provide a valuable summary of 
results across many studies in the field of reward processing in addiction. We acclaim this study and would like to contribute to the discussion about the implications of the findings by pointing out some of the issues that need to be addressed in future research to further advance the field.

Inevitably, studies brought together in a meta-analysis differ, but when a group of studies is sufficiently homogeneous, the results are thought to be robust. In the article, ${ }^{1}$ the substance use disorder (SUD) section primarily included studies using the monetary incentive delay task (13 of 17 studies), adding to the robustness of the SUD results. However, there is considerable heterogeneity in the tasks included in the gambling disorder (GD) section: only 4 of 8 studies used the monetary incentive delay task. Other studies focused on expectancy value coding, ${ }^{2}$ effort/motivation-related activity, ${ }^{3}$ reversal learning, ${ }^{4}$ and blackjack-related wins and losses. ${ }^{5}$ Thus, although these tasks measured aspects of reward processing, not all of them were specifically tailored to capture reward anticipation and outcome notification. Additionally, the fMRI contrasts included in the meta-analysis ${ }^{1}$ were not necessarily the contrasts of main interest of these studies. Together, this may have resulted in reduced power for the included contrasts and activation of brain areas (eg, dorsal vs ventral striatum) associated with other aspects of reward processing assessed in these tasks. Therefore, the results concerning the GD studies should be interpreted with caution, and any conclusion about differences between SUD and GD is possibly confounded. Moreover, although the authors ${ }^{1}$ alluded to disparate patterns of brain activation between SUD and GD, this was not statistically tested.

All things considered, the conclusion that the differences between SUD and GD are due to the monetary nature of the rewards is not substantiated at this point. More monetary incentive delay studies in GD will provide consistency in future meta-analyses and will facilitate comparisons with SUD. We endorse the authors' recommendation to "go beyond the use of monetary rewards in fMRI studies, eg, using positive scenes, food, juice, or erotic stimuli," with the ultimate goal to univocally arbitrate between theories of addiction.

Ruth J. van Holst, PhD

Tim van Timmeren, Msc

Anneke E. Goudriaan, PhD

Author Affiliations: Academic Medical Center, Department of Psychiatry, University of Amsterdam, Amsterdam, the Netherlands.

Corresponding Author: Ruth J. van Holst, PhD, Academic Medical Center, Department of Psychiatry, University of Amsterdam, Meibergdreef 5 , Amsterdam 1100 DD, the Netherlands (mail@ruthvanholst.nl).

Published Online: June 7, 2017. doi:10.1001/jamapsychiatry.2017.1051 Conflict of Interest Disclosures: None reported.

1. Luijten M, Schellekens AF, Kühn S, Machielse MWJ, Sescousse G. Disruption of reward processing in addiction: an image-based meta-analysis of functional magnetic resonance imaging studies. JAMA Psychiatry. 2017;74(4):387-398.

2. van Holst RJ, Veltman DJ, Büchel C, van den Brink W, Goudriaan AE. Distorted expectancy coding in problem gambling: is the addictive in the anticipation? Biol Psychiatry. 2012;71(8):741-748.

3. Fauth-Bühler M, Zois E, Vollstädt-Klein S, Lemenager T, Beutel M, Mann K. Insula and striatum activity in effort-related monetary reward processing in gambling disorder: the role of depressive symptomatology. Neuroimage Clin. 2014;6:243-251.
4. de Ruiter MB, Oosterlaan J, Veltman DJ, van den Brink W, Goudriaan AE. Similar hyporesponsiveness of the dorsomedial prefrontal cortex in problem gamblers and heavy smokers during an inhibitory control task. Drug Alcohol Depend. 2012;121(1-2):81-89.

5. Miedl SF, Fehr T, Meyer G, Herrmann M. Neurobiological correlates of problem gambling in a quasi-realistic blackjack scenario as revealed by fMRI. Psychiatry Res. 2010;181(3):165-173.

In Reply Van Holst et al provide a thoughtful comment on our meta-analysis of functional magnetic resonance imaging studies investigating reward processing in addiction. ${ }^{1}$ Their letter mainly focuses on 2 methodological points.

First, van Holst et al highlight that the tasks used in gambling disorder (GD) studies are less homogeneous than those in substance use disorder (SUD) studies. Although this is correct-and reflects fewer studies available on GD-we believe that contrast homogeneity, rather than task homogeneity, is what primarily matters. In keeping with this, we carefully selected those contrasts that capture the 2 cognitive processes of interest in our meta-analysis, namely monetary reward anticipation and outcome. Incidentally, this is the reason why some of these contrasts depart from those reported in the original articles. It is worth emphasizing that heterogeneity is a pervasive problem in (neuroimaging) meta-analyses that extends beyond tasks and includes heterogeneity in design efficiency, data analysis, and population selection. However, task heterogeneity is not necessarily detrimental, as the convergence of activations despite such heterogeneity ensures that the results are not due to idiosyncrasies in task design and are generalizable across a variety of paradigms. ${ }^{2}$

Second, as van Holst et al correctly point out, the SUD and GD populations were not directly compared. The main reason is that these populations originated from different studies and were thus unlikely to be matched. In addition, our rationale for including both SUD and GD studies was the search for similarities, rather than differences, in brain activations. As a result, we agree with van Holst et al that differences uncovered by our meta-analysis between brain maps for SUD and GD studies are inherently qualitative and should be interpreted with caution.

Yet, it is of crucial relevance to explore neurobiological similarities and differences between behavioral addictions, such as GD, and substance addictions because this can shed light on several highly debated issues. First, there is a growing number of problematic behaviors being regarded as potential behavioral addictions including problematic internet use, binge eating, compulsive buying, and compulsive sexual activities. ${ }^{3}$ Accordingly, in the DSM-5, a chapter on substance use and addictive disorders was included containing GD as a behavioral addiction and internet gaming disorder as a "condition for further study." Unraveling the neural mechanisms underlying these problematic behaviors could help refine the boundaries and definition of behavioral addictions. Second, substance and behavioral addictions have partly similar diagnostic characteristics, such as craving, diminished behavioral control, tolerance, and withdrawal-like symptoms. It is unclear whether this homogeneity in symptoms also reflects shared neurobiological mechanisms. This question is clinically relevant, as treatment approaches for SUD are currently adapted and applied to behavioral 
addictions including GD. ${ }^{4,5}$ Neuroimaging studies could shed light on the sensibility of such an approach and could potentially guide future treatment development for substance and behavioral addictions.

In conclusion, we fully agree with van Holst et al that more studies investigating reward processing in behavioral addictions are needed to further advance our understanding of shared and distinct neural mechanisms contributing to SUD and behavioral addictions.

\section{Arnt F. Schellekens, MD, PhD \\ Maartje Luijten, PhD \\ Guillaume Sescousse, $\mathrm{PhD}$}

Author Affiliations: Radboud University Medical Centre, Department of Psychiatry, Nijmegen Institute for Scientist Practitioners in Addiction, Nijmegen, the Netherlands (Schellekens); Behavioural Science Institute, Radboud University, Nijmegen, the Netherlands (Luijten); Donders Institute for Brain, Cognition and Behaviour, Radboud University, Nijmegen, the Netherlands (Sescousse).

Corresponding Author: Maartje Luijten, PhD, Behavioural Science Institute, Radboud University, PO Box 9104, Room A06.19a, 6500 HE, Nijmegen, the Netherlands (m.luijten@bsi.ru.nl).

Published Online: June 7, 2017. doi:10.1001/jamapsychiatry.2017.1054

Conflict of Interest Disclosures: None reported.

1. Luijten M, Schellekens AF, Kühn S, Machielse MW, Sescousse G. Disruption of reward processing in addiction: an image-based meta-analysis of functional magnetic resonance imaging studies. JAMA Psychiatry. 2017;74(4):387-398.

2. Costafreda SG. Pooling FMRI data: meta-analysis, mega-analysis and multi-center studies. Front Neuroinform. 2009;3:33.

3. Kardefelt-Winther D, Heeren A, Schimmenti A, et al. How can we conceptualize behavioural addiction without pathologizing common behaviours? Addiction. 2017.

4. Cowlishaw S, Merkouris S, Dowling N, Anderson C, Jackson A, Thomas S. Psychological therapies for pathological and problem gambling. Cochrane Database Syst Rev. 2012;11:CD008937.

5. Grant JE, Odlaug BL, Schreiber LRN. Pharmacological treatments in pathological gambling. Br J Clin Pharmacol. 2014;77(2):375-381.

\section{First-Episode Schizophrenia and Diabetes Risk}

To the Editor In their article in JAMA Psychiatry, Pillinger and colleagues ${ }^{1}$ shed light on the association between schizophrenia and an increased risk for type 2 diabetes, showing that patients are at increased risk for diabetes from the onset of illness and not only as a consequence of long-term treatment and chronic illness. But these data also beg the question as to why there is abnormal glucose metabolism and bioenergetics in the disorder.

The first indication of abnormal energy generation in schizophrenia, increased lactate and decreased glutathione, was noted as early as $1934 .^{2}$ Mitochondrial function is now confirmed to be abnormal in schizophrenia and drives part of the pathophysiology of the disorder as well as comorbidities such as glucose dysregulation. Numerous molecular functional neuroimaging studies have connected compromised brain energy metabolism and oxidative stress due to mitochondrial dysfunction with the pathophysiology of schizophrenia. ${ }^{2}$

Type 2 diabetes is a complex metabolic disease due to pancreatic $\beta$-cell dysfunction and is associated with obesity, insulin resistance, and hypoinsulinemia. Impaired glucose homeostasis is associated with compromised mitochondrial biogenesis and function. ${ }^{3}$
Because long-term antipsychotic use increases the risk for type 2 diabetes, this is a confounding factor in the association of impaired glucose homeostasis and schizophrenia. Being able to remove this bias by analyzing individuals with firstepisode schizophrenia allows observation of early alterations in glucose homeostasis in this subgroup. This finding of abnormal glucose homeostasis at disease onset supports the concept that bioenergetic dysregulation driven by mitochondrial dysfunction may represent a common molecular mechanism that underpins both schizophrenia and type 2 diabetes, and explains, at least in part, their frequent co-occurrence.

In summary, Pillinger and colleagues' study ${ }^{1}$ creates an imperative for new research to determine the molecular mechanism(s) defining the potentially overlapping relationship between schizophrenia and type 2 diabetes. Mitochondrial dysfunction is a logical target. The mechanistic roads to mitochondrial dysfunction include other biological pathways that are linked to diabetes, schizophrenia, and other comorbid disorders. ${ }^{4}$ Defining the molecular pathways that lead to mitochondrial dysfunction has the potential to enhance our understanding of the underlying pathophysiology of schizophrenia and potentially define novel treatment targets.

Chiara C. Bortolasci, PhD

Michael Berk, MD, PhD

Ken Walder, $\mathrm{PhD}$

Author Affiliations: Centre for Molecular and Medical Research, School of Medicine, Deakin University, Geelong, Victoria, Australia (Bortolasci, Walder); IMPACT Strategic Research Centre, School of Medicine, Deakin University, Geelong, Victoria, Australia (Berk).

Corresponding Author: Chiara C. Bortolasci, PhD, Centre for Molecular and Medical Research, School of Medicine, Deakin University, 75 Pigdons Rd, Waurn Ponds, Geelong, Victoria 3216, Australia (bchiara@deakin.edu.au).

Published Online: May 24, 2017. doi:10.1001/jamapsychiatry.2017.0760 Conflict of Interest Disclosures: None reported.

1. Pillinger T, Beck K, Gobjila C, Donocik JG, Jauhar S, Howes OD. Impaired glucose homeostasis in first-episode schizophrenia: a systematic review and meta-analysis. JAMA Psychiatry. 2017;74(3):261-269.

2. Rajasekaran A, Venkatasubramanian G, Berk M, Debnath M. Mitochondrial dysfunction in schizophrenia: pathways, mechanisms and implications. Neurosci Biobehav Rev. 2015;48:10-21.

3. Martin SD, McGee SL. The role of mitochondria in the aetiology of insulin resistance and type 2 diabetes. Biochim Biophys Acta. 2014;1840(4):1303-1312.

4. Morris G, Berk M. The many roads to mitochondrial dysfunction in neuroimmune and neuropsychiatric disorders. BMC Med. 2015;13:68.

To the Editor In their recent systematic review and metaanalysis, Pillinger and colleagues ${ }^{1}$ examined whether individuals with first-episode schizophrenia exhibit an "inherent risk" for type 2 diabetes. Their study has several limitations, most notably the methodological limitations of the studies contributing to the meta-analysis as well as an overinterpretation of results.

The studies included in the meta-analysis appear to be convenience samples with small sample sizes (10-120 patients), rather than representative or random samples that could protect against sampling bias. Despite no prior evidence for de novo $\beta$-cell failure or ketoacidosis in antipsychotic-naive patients, the analysis focused on diabetes diagnostic criteria 